\title{
Tag Return Models Allowing for Harvest and Catch and Release: Evidence of Environmental and Management Impacts on Striped Bass Fishing and Natural Mortality Rates
}

\author{
HONGHUA JiANG \\ Department of Statistics, North Carolina State University, Raleigh, North Carolina 27695, USA \\ KenNeth H. PollocK* \\ Department of Zoology, North Carolina State University, Raleigh, North Carolina 27695, USA \\ Cavell Brownie \\ Department of Statistics, North Carolina State University, Raleigh, North Carolina 27695, USA \\ John M. Hoenig and Robert J. Latour \\ Virginia Institute of Marine Science, College of William and Mary, \\ Post Office Box 1346, Gloucester Point, Virginia 23062, USA \\ BRIAN K. WELLS \\ National Oceanic and Atmospheric Administration, National Marine Fisheries Service, \\ 110 Shaffer Road, Santa Cruz, California 95060, USA \\ JosePh E. Hightower \\ U.S. Geological Survey, North Carolina Cooperative Fish and Wildlife Research Unit, \\ Department of Zoology, North Carolina State University, Raleigh, North Carolina 27695, USA
}

\begin{abstract}
Catch-and-release fisheries have become very important in the management of overexploited recreational fish stocks. Tag return studies, where the tag is removed regardless of fish disposition, have been used to assess the effectiveness of restoration efforts for these fisheries. We extend the instantaneous rate formulation of tag return models to allow for catch and release as well as harvest. The key point of our methods is that, given an estimate of the tag reporting rate, the fishing mortality rate $(F)$ is separated into two components: the mortality on harvested fish and the "mortality" on tags (because the tags are removed) of fish released alive. The total fishing mortality rate for untagged fish is the sum of the $F$ s due to harvest and hooking mortality suffered by fish released alive. Natural mortality rates can also be estimated. Both ageindependent models and age-dependent models are constructed, and the age-dependent models are illustrated by application to data from a study of striped bass Morone saxatilis in Chesapeake Bay from 1991 to 2003 by the Maryland Department of Natural Resources. By fitting models of the natural mortality rate with limited age and year dependence, we demonstrate an overall decrease in natural mortality rates as fish age and provide evidence of an increase in natural mortality beginning in the late 1990s, when an outbreak of the disease mycobacteriosis is thought to have begun. Our results indicate that fishing mortality is age dependent; selectivity increases up to age 6 , when fish appear to be fully recruited to the fishery. There is also evidence of an increase in fishing mortality since 1995, when regulations were relaxed.
\end{abstract}

In traditional fisheries tag return studies (e.g., Hoenig et al. 1998a, 1998b), all caught fish are assumed to be harvested. However, many present-day fisheries include substantial catch-and-release fishing, so that only a fraction of the captured fish are harvested while the remainder are released alive. The goal is to

\footnotetext{
* Corresponding author: pollock@unity.ncsu.edu
}

Received March 19, 2006; accepted July 25, 2006 Published online March 29, 2007 provide recreational fishing opportunities while conserving the stock. Catch-and-release fisheries have become very important to the management of overexploited recreational fish stocks. However, little work has been done on how to estimate population demographic parameters (such as fishing and natural mortality) for catch-and-release fisheries using tagging studies. Estimation methods that account for catch-andrelease fishing are essential in order to assess the effectiveness of conservation efforts.

The probability of a tag's being reported may be 
different for a released fish than it is for a harvested fish. In some fisheries, the tags are removed from the fish and then reported (i.e., returned to the agency), while in others the tag numbers are recorded and reported to the agency without the tags' being removed. There are advantages and disadvantages to both approaches. If tags are removed and returned to the agency, there should be few errors in recording tag numbers. However, no additional data can be obtained from those fish because they are now unmarked. If tag numbers are recorded and the fish released with the tags intact, the chance of recording errors could be substantial. On the other hand, these fish remain marked and could be recaptured multiple times, providing additional information on survival. The latter can be viewed as a generalized type of Jolly-Seber model (Seber 1982; Williams et al. 2002).

The method used to estimate the tag reporting rate also may affect the decision to remove the tag before releasing a fish. If a high-reward tagging approach is used to estimate the regular tag reporting rate (Pollock et al. 2001), the tags would need to be removed and returned because agencies would require having the tag in hand to pay the reward. However, if the angler survey method is used to estimate the regular tag reporting rate (Pollock et al. 1991), tags could either be cut off or left on the fish provided that the agent got a report of the released tag numbers. Here we focus on the situation in which the tag is removed at capture whether the fish is kept or released, as this approach has been used in several studies of Atlantic striped bass Morone saxatilis.

Smith et al. (2000) presented a method of accounting for catch-and-release fishing in the estimation of total and fishing mortality. Their likelihood was based on finite recovery and survival rates, in which the survival rate for tags was represented as the survival rate for fish with an adjustment for catch-and-release fishing. This ad hoc adjustment involved parameters for the shortterm mortality after release (i.e., hooking mortality) and the tag reporting rate, $\lambda$. Assuming known values for the instantaneous natural mortality, $M(0.15)$, and hooking mortality (0.09), an iterative process was used to obtain estimates of the reporting rate, fishing mortality, and survival that accounted for catch-andrelease fishing. We develop an alternative probability model and likelihood for the tag return data that is simpler and more intuitive. We use the methodology developed to analyze data from a tagging study carried out by the Maryland Department of Natural Resources. We conclude with a discussion of the important issues raised, including important model assumptions, as well as suggestions for future research.

\section{Age-Independent Models}

The key point in modeling tag returns from catchand-release fishing is to note that removing tags from fish that are caught and released leads to an additional source of "mortality" on the tags that is not necessarily experienced by the fish. Jiang (2005) assumed that tag returns were recorded separately for fish that were harvested and those that were caught and released and developed a generalization of the Hoenig et al. (1998a) instantaneous rates models. Here we present the key elements of this approach. We first consider the situation where tagged fish are assumed to be fully recruited and all rates can be considered age independent and later present age-dependent versions of the method.

\section{Glossary of Terms}

We define the following variables:

$N_{i}=$ the number of adult fish tagged and released in year $i(i=1,2, \ldots, I)$;

$R_{i j}=$ the number of these $N_{i}$ fish that are subsequently harvested and reported in year $j(j=\mathrm{i}$, $i+1, i+2, \ldots, J)$;

$R_{i j}^{\prime}=$ the number of these $N_{i}$ fish that are caught, released without a tag, and reported in year $j$;

$F_{j}=$ the instantaneous rate of fishing mortality on fish in year $j$;

$F_{j}^{\prime}=$ the instantaneous rate of fishing mortality in year $j$ on the tags taken from fish that are caught and released;

$M=$ the instantaneous rate of natural mortality;

$P_{i j}=$ the probability that a fish tagged and released in year $i$ will be harvested and its tag reported in year $j$;

$P_{i j}^{\prime}=$ the probability that a fish tagged and released in year $i$ will be caught and released and its tag reported in year $j$;

$S_{j}=$ the annual survival rate in year $j$ for tags on fish alive at the beginning of year $j$;

$\lambda=$ the tag reporting rate (the probability that the tag will be reported) given that a tagged fish is harvested;

$\lambda^{\prime}=$ the tag reporting rate (the probability that the tag will be reported) given that a tagged fish is recaptured, the tag is clipped off, and the fish is released alive.

To reduce the problems associated with the nearsingularity of the models (Jiang 2005), we had to assume that the tag reporting rates $\lambda$ and $\lambda^{\prime}$ were equal and constant over years. (If information is available on how $\lambda$ and $\lambda^{\prime}$ differ and how they vary over time, the models can be modified in a straightforward manner.) 
Similarly, in the basic model the natural mortality rate $M$ was assumed to be constant over years. All parameters are age independent, although extensions to multiage models (with age- and year-dependent $M \mathrm{~s}$ ) are presented in a later section.

\section{Model Development}

The expected number of tag returns from fish tagged and released in year $i$ and harvested in year $j$ is

$$
E\left(R_{i j}\right)=N_{i} P_{i j}
$$

where

$$
\begin{aligned}
& P_{i j}= \begin{cases}\left(\prod_{v=i}^{j-1} S_{v}\right)\left(1-S_{j}\right) \frac{F_{j}}{F_{j}+F_{j}^{\prime}+M} \lambda & (\text { when } j>i) \\
\left(1-S_{j}\right) \frac{F_{j}}{F_{j}+F_{j}^{\prime}+M} \lambda & (\text { when } j=i)\end{cases} \\
& S_{j}=\exp \left(-F_{j}-F_{j}^{\prime}-M\right) .
\end{aligned}
$$

The expected number of tag returns from fish tagged and released in year $i$ and recaptured and released without a tag in year $j$ is

$$
E\left(R_{i j}^{\prime}\right)=N_{i} P_{i j}^{\prime},
$$

where

$$
\begin{aligned}
& P_{i j}^{\prime}= \begin{cases}\left(\prod_{v=i}^{j-1} S_{v}\right)\left(1-S_{j}\right) \frac{F_{j}^{\prime}}{F_{j}+F_{j}^{\prime}+M} \lambda^{\prime} & (\text { when } j>i) \\
\left(1-S_{j}\right) \frac{F_{j}^{\prime}}{F_{j}+F_{j}^{\prime}+M} \lambda^{\prime} & (\text { when } j=i)\end{cases} \\
& S_{j}=\exp \left(-F_{j}-F_{j}^{\prime}-M\right) .
\end{aligned}
$$

For the batch of $N_{i}$ fish tagged and released in year $i$, assuming independent fates, the tag returns in subsequent years, $R_{i j}$ and $R_{i j}^{\prime}$, follow a multinomial distribution. Therefore, the full likelihood function is product multinomial following Hoenig et al. (1998a), that is,

$$
\begin{aligned}
L= & \prod_{i=1}^{I}\left[\begin{array}{c}
N_{i} \\
R_{i i}, R_{i i+1}, \ldots, R_{i J}, R_{i i}^{\prime}, R_{i i+1}^{\prime}, \ldots, R_{i J}^{\prime}, N_{i} \\
-\sum_{j=i}^{J}\left(R_{i j}+R_{i j}^{\prime}\right)
\end{array}\right] \\
& \times\left(\prod_{j=i}^{J} P_{i j}^{R_{i j}} P_{i j}^{\prime R_{i j}^{\prime}}\right) \\
& \times\left[1-\sum_{v=i}^{J}\left(P_{i v}+P_{i v}^{\prime}\right)\right]^{N_{i}-\Sigma_{v=i}^{J}\left(R_{i v}+R_{i v}^{\prime}\right)} .
\end{aligned}
$$

Theoretically, maximum likelihood estimators of the instantaneous rates $F_{j}, F_{j}^{\prime}(j=1,2, \ldots, J)$, and $M$ and the tag reporting rates $\lambda$ and $\lambda^{\prime}$ can be obtained from this likelihood using software such as program SURVIV (White 1983). Total annual mortality rates for fish then can be estimated from the instantaneous rates. However, it is often very difficult to obtain good estimates of the tag reporting rates in addition to the other parameters. If reliable values of the tag reporting rates are available from other sources, such as a highreward tagging study, these parameters can be treated as known and estimates obtained for $F_{j}, F_{j}^{\prime}(j=1,2$, $\ldots, J$ ) and $M$. An even better approach, if data for estimating the reporting rate are available (e.g., from a reward tagging study), is to include an additional component in the likelihood function and estimate all parameters, including $\lambda$ and $\lambda^{\prime}$, together (see Hoenig et al. 1998a).

The total instantaneous mortality for tags $\left(Z_{j, \text { tags }}\right)$ and the annual survival rate for tags $\left(S_{j, \text { tags }}\right)$ in year $j$ are

$$
Z_{j, \text { tags }}=F_{j}+F_{j}^{\prime}+M
$$

and

$$
S_{j, \text { tags }}=\exp \left(-F_{j}-F_{j}^{\prime}-M\right) .
$$

Following the approach in Brooks et al. (1998), the total annual exploitation rate for tags, $U_{j, \text { tags }}$, has two components depending upon whether the fish is kept (i.e., harvested) or released. Thus

$$
U_{j, \text { tags }}=U_{j, \text { tags }}(\text { kept })+U_{j, \text { tags }}(\text { rels }),
$$

where

$$
\begin{aligned}
& U_{j, \text { tags }}(\mathrm{kept}) \\
& \quad=\frac{F_{j}}{F_{j}+F_{j}^{\prime}+M}\left\{1-\exp \left[-\left(F_{j}+F_{j}^{\prime}+M\right)\right]\right\},
\end{aligned}
$$

and

$$
\begin{aligned}
& U_{j, \text { tags }}(\text { rels }) \\
& \quad=\frac{F_{j}^{\prime}}{F_{j}+F_{j}^{\prime}+M}\left\{1-\exp \left[-\left(F_{j}+F_{j}^{\prime}+M\right)\right]\right\} .
\end{aligned}
$$

To obtain the corresponding rates for fish, we must know to what extent fish that are caught and released are subject to hooking mortality, $\delta$ (the mortality immediately following release attributable to hooking and other handling stresses). To account for hooking mortality on the fish, we let the product $\delta \cdot F^{\prime}$ represent the instantaneous force of mortality on caught-andreleased fish, where $0 \leq \delta \leq 1$. The annual survival rate and total instantaneous mortality of fish are then

$$
S_{j, \text { fish }}=\exp \left[-\left(F_{j}+\delta \cdot F_{j}^{\prime}+M\right)\right]
$$

and

$$
Z_{j, \text { fish }}=F_{j}+\delta \cdot F_{j}^{\prime}+M
$$

If all the released fish die after release because of 
hooking mortality, $\delta=1$ and the survival and mortality rates for fish are the same as those for tags, namely,

$$
S_{j, \text { fish }}=\exp \left[-\left(F_{j}+F_{j}^{\prime}+M\right)\right]
$$

and

$$
Z_{j, \text { fish }}=F_{j}+F_{j}^{\prime}+M .
$$

At the other extreme, assuming none of the released fish die, $\delta=0$ and

$$
S_{j, \text { fish }}=\exp \left[-\left(F_{j}+M\right)\right]
$$

and

$$
Z_{j, \text { fish }}=F_{j}+M \text {. }
$$

Diodati and Richards (1996) conducted an experiment to estimate the hooking mortality on striped bass. They found that many factors influence hooking mortality, including hook penetration, gear type, and angler experience. The water temperatures in their study did not exceed $25^{\circ} \mathrm{C}$ and dissolved oxygen was above critical levels, so they did not find significant effects of temperature on hooking mortality. They reported that the estimated hooking mortality was $9 \%$ $(\mathrm{SE}=2 \%)$. This estimate of hooking mortality is a finite rate, but it can be used as an approximation to the hooking mortality rate, $\delta$, in our instantaneous rates formulation. The proof for this approximation is given in Jiang (2005). In Jiang (2005), an age-independent example of the analysis is presented for striped bass. It included use of the $9 \%$ hooking mortality rate to obtain adjusted total mortality rates. However, in the interests of brevity, we here move immediately to the agedependent model development and then illustrate the methodology with the striped bass analysis allowing age dependence of fishing and natural mortality rates.

\section{Age-Dependent Models}

Jiang (2005) presented a likelihood function for data from traditional multiple-age tagging studies, where all the recaptured fish were harvested. Merging that approach with the work in the previous section, we present a likelihood function for data from multiple-age tagging studies on fisheries that include both harvest and catch and release.

\section{Glossary of Terms}

We extend the notation in the previous section by including an additional subscript $k$ to denote age at tagging:

$N_{i k}=$ the number of fish tagged at age $k(k=1,2, \ldots$, $K)$ and released in year $i(i=1,2, \ldots, I)$;

$R_{i j k}=$ the number of these $N_{i k}$ fish that are subsequently harvested and reported in year $j$;

$R_{i j k}^{\prime}=$ the number of these $N_{i k}$ fish that are caught, reported, and released without a tag in year $j(j$ $=i, i+1, i+2, \ldots, J)$.

We assume that the components of fishing mortality on tags attached to fish of age $a$ in year $j$ are

$$
F_{j a}=\operatorname{Sel}_{a} F_{j}
$$

and

$$
F_{j a}^{\prime}=\operatorname{Sel}_{a} F_{j}^{\prime} ;
$$

$F_{j}=$ the instantaneous rate of fishing mortality for fully recruited fish that are harvested;

$F_{j}^{\prime}=$ the instantaneous rate of fishing mortality on the tags for fully recruited fish that are released alive;

Sel $_{a}=$ the selectivity coefficient for age- $a$ fish at the time of recovery.

Selectivity is assumed to be constant over years for each age and to be 1 for all fish above a certain age $a_{c}$ (Sel ${ }_{a} \equiv 1$ for $a \geq a_{c}$ ). Relating age at tagging $(k)$ to age at recovery $(a)$, we have $a=k+j-i$, which is used in the expressions that follow. Here the parameters $\lambda$ and $\lambda^{\prime}$ are assumed to be age independent because we do not know of practical cases where age-specific reporting rates have been estimated. If such data became available we could easily extend the models to allow the age dependence of reporting rates. For $P_{i j k}$, $P_{i j k}^{\prime}$, and $S_{i j k}$ the subscript $k$ indicates the age at tagging. In the basic model, we assume that the natural mortality rate $M$ is constant over ages and years.

\section{Model Development}

The expected number of tag returns from fish tagged and released at age $k$ in year $i$, then harvested in year $j$, is

$$
E\left(R_{i j k}\right)=N_{i k} P_{i j k}
$$

where

$$
P_{i j k}=\left\{\begin{array}{c}
\left(\prod_{v=i}^{j-1} S_{i v k}\right)\left(1-S_{i j k}\right) \frac{F_{j} \operatorname{Sel}_{k+j-i}}{\left(F_{j}^{\prime}+F_{j}\right) \operatorname{Sel}_{k+j-i}+M} \lambda \\
(\text { when } j>i) \\
\left(1-S_{i j k}\right) \frac{F_{j} \operatorname{Sel}_{k}}{\left(F_{j}^{\prime}+F_{j}\right) \operatorname{Sel}_{k}+M} \lambda \\
\left.(\text { when } j=i)^{\prime}=\operatorname{Sel}_{k+j-1}-M\right] .
\end{array}\right.
$$

The expected number of tag returns from fish tagged and released at age $k$ in year $i$, then recaptured and released without the tag in year $j$, is

$$
E\left(R_{i j k}^{\prime}\right)=N_{i k} P_{i j k}^{\prime}
$$

where 


$$
\begin{aligned}
& \int\left(\prod_{v=i}^{j-1} S_{i v k}\right)\left(1-S_{i j k}\right) \frac{F_{j}^{\prime} \operatorname{Sel}_{k+j-i}}{\left(F_{j}^{\prime}+F_{j}\right) \operatorname{Sel}_{k+j-i}+M} \lambda^{\prime} \\
& P_{i j k}^{\prime}=\left\{\begin{array}{c}
(\text { when } j>i) \\
\left(1-S_{i j k}\right) \frac{F_{j}^{\prime} \operatorname{Sel}_{k}}{\left(F_{j}^{\prime}+F_{j}\right) \operatorname{Sel}_{k}+M} \lambda^{\prime} \\
(\text { when } j=i)
\end{array}\right. \\
& S_{i j k}=\exp \left[-\left(F_{j}+F_{j}^{\prime}\right) \operatorname{Sel}_{k+j-1}-M\right] \text {. }
\end{aligned}
$$

For those $N_{i k}$ fish that are tagged at age $k$ and released in year $i$, the tag returns in subsequent years, $R_{i j k}$ and $R_{i j k}^{\prime}$, follow a multinomial distribution. The full likelihood function is the product multinomial

$$
\begin{aligned}
L= & \prod_{k=1}^{K} \prod_{i=1}^{I}\left[\begin{array}{c}
N_{i k} \\
R_{i i k}, R_{i i+1 k}, \ldots, R_{i J k}, R_{i i k}^{\prime}, R_{i i+1 k}^{\prime}, \ldots, \\
R_{i J k}^{\prime}, N_{i k}-\sum_{j=i}^{J}\left(R_{i j k}+R_{i j k}^{\prime}\right)
\end{array}\right] \\
& \times\left(\prod_{j=i}^{J} P_{i j k}^{R_{i j k}} P_{i j k}^{\prime} R_{i j k}^{\prime}\right) \\
& \times\left[1-\sum_{v=i}^{J}\left(P_{i v k}+P_{i v k}^{\prime}\right)\right]^{N_{i k}-\Sigma_{v=i}^{J}\left(R_{i v k}+R_{i v k}^{\prime}\right)} .
\end{aligned}
$$

We use the method of maximum likelihood to estimate $F_{j}, F_{j}^{\prime}(j=1,2, \ldots, J), M$, selectivities $\operatorname{Sel}_{a}(a=1, \ldots$, $\left.a_{c}-1\right)$, and the tag reporting rates $\lambda$ and $\lambda^{\prime}$. If the tag reporting rates are known, estimates are obtained for the remaining variables. Owing to their important biological interest, we also investigate models in which $M$ is year and/or age dependent, but we do this only to a limited extent to avoid parameter identifiability problems. Following the same approach we took for the age-independent mortality model, we could estimate total mortality allowing for hooking mortality.

\section{Example}

\section{Study Description}

We illustrate the analysis of age-dependent tag return data from a fishery with both harvest and catch and release using 1991-2003 Maryland Department of Natural Resources data for striped bass (Appendix A in Jiang 2005). Over 24,533 striped bass were marked (March through June) with an internal anchor tag (Floy tag FM-84) and released in the upper Chesapeake Bay. A scale-based aging method was used to identify the ages of approximately $30 \%$ of the tagged fish; the remaining fish were aged based on length bins. This resulted in seven age-groups (from age 2 to age $8+$ ). Scales work reliably for striped bass up to age 8 , and there is no need for an otolith-based method (Secor et al. 1995). Tag returns from fish at large for less than 1 week were excluded to ensure reasonable mixing. We only analyzed the data for striped bass classified as age 3 and above (24,356 total fish) because the sample sizes for those marked at age 2 were too small. A total of 4,593 tags were returned to fishery agencies, representing 2,960 (64\%) fish that were harvested (killed) and 1,633 (36\%) that were released alive after the tag was removed.

The effects of age on the fishing mortality of harvested fish $(F)$ and on the tags of fish that were caught and released $\left(F^{\prime}\right)$ were incorporated through selectivity. Selectivity was estimated separately for striped bass of ages 3, 4, and $5\left(\mathrm{Sel}_{3}, \mathrm{Sel}_{4}\right.$, and $\left.\mathrm{Sel}_{5}\right)$. Selectivity was assumed to be 1 for fish age 6 and older because in preliminary runs of our models in which we estimated selectivity we found it to be 1 for age- 6 fish. We considered the basic model $\left(F_{y}, F_{y}^{\prime}, M, \mathrm{Sel}_{3}, \mathrm{Sel}_{4}\right.$, $\mathrm{Sel}_{5}$ ), in which $F$ and $F^{\prime}$ were age dependent through selectivity and allowed to vary by year (subscript $y$ ) and $M$ was constant over years and ages. Tag shedding and tag-induced mortality were assumed to be negligible based on special studies (following Smith et al. 2000), and we assumed $\lambda=\lambda^{\prime}=0.43$. This value is based on the results of a high-reward tagging study conducted by the Delaware Division of Fish and Wildlife and is the average reporting rate for 1991 and 1997 estimated in Smith et al. (2000; range, 0.38$0.48)$. We also fit the corresponding model $\left(F_{y}, F_{y}^{\prime}, M\right.$, $\left.\mathrm{Sel}_{3}, \mathrm{Sel}_{4}, \mathrm{Sel}_{5}, \lambda, \lambda^{\prime}\right)$, where $\lambda$ and $\lambda^{\prime}$ are estimated.

To investigate the assumption that natural mortality is constant over ages, we fit a model $\left(F_{y}, F_{y}^{\prime}, M_{Y}, M_{A}\right.$, $\mathrm{Sel}_{3}, \mathrm{Sel}_{4}, \mathrm{Sel}_{5}$ ), where we assumed that young $(Y)$ fish of ages 3,4 , and 5 had a common natural mortality rate different from that of adult $(A)$ fish of ages 6 and above. We also fit the model $\left(F_{y}, F_{y}^{\prime}, M_{3}, M_{4}, M_{5}, M_{A}\right.$, $\mathrm{Sel}_{3}, \mathrm{Sel}_{4}, \mathrm{Sel}_{5}$ ), which allowed fish of ages 3,4 , and 5 to have different natural mortality rates. The corresponding models in which reporting rates $\lambda$ and $\lambda^{\prime}$ were estimated were also investigated.

It was of special interest to investigate models that allow the natural mortality rate to differ with time because of possible disease effects. A bacterial disease known as mycobacteriosis, which is caused by bacteria in the genus Mycobacterium, appeared in Chesapeake Bay striped bass in the late 1990s (Cardinal 2001). Symptoms include external lesions (open sores on the skin) and internal lesions that look like lumps in the spleen and kidney. There are about a dozen species of mycobacteria in striped bass and it is not known which species cause the disease or diseases. The prevalence of mycobacteriosis in striped bass may be $60 \%$ or even higher in the Rappahannock River, Virginia, in late summer (Cardinal 2001). Owing to concerns about 
parameter redundancy, we assumed that natural mortality was constant in the years before the disease effects occurred (1999) and constant at a different value after they began. Finally, reduced models that reflected the effects of the relaxation of fishing regulations on fishing mortality in 1995 (Richards and Rago 1999) were also investigated.

We used Akaike's information criterion (AIC) to choose between models because this method is able to compare multiple nested and nonnested models. Akaike's information criteria is a statistic that deals with the tradeoff between the reduced bias associated with having more parameters and the smaller estimator variance associated with having fewer parameters (Burnham and Anderson 2002). It can be computed as

$$
\mathrm{AIC}=-2 \log [l(\underline{\hat{\theta}} \mid y)]+2 k,
$$

where $\log [l(\underline{\hat{\theta}} \mid y)]$ is the $\log$ likelihood function evaluated at the maximum likelihood estimator values of $\underline{\hat{\theta}}$ given the data $y$ and $k$ is the number of parameters. The model with the minimum AIC value was selected.

Overdispersion in the data can result from a lack of independence between capture and survival events, which stems from the fact that fish travel in schools. If overdispersion is the reason for the lack of fit in a model, a quasilikehood approach is recommended (Burnham and Anderson 2002); that was the approach we followed in this paper. The corresponding criterion is QAIC, defined as

$$
\text { QAIC }=-2 \log [l(\underline{\hat{\theta}} \mid y)] / \hat{c}+2 k,
$$

where $\hat{\mathrm{c}}$ is a variance inflation factor that can be calculated as

$$
\hat{c}=\chi^{2} / \mathrm{df} ;
$$

$\chi^{2}$ and df correspond to the value of the Pearson goodness-of-fit test of the most general model in the model set and its degrees of freedom. The small-sample correction to AIC and QAIC (Burnham and Anderson 2002) made no difference for this example and is not reported.

\section{Results}

All models with tag reporting rates fixed at 0.43 produced parameter estimates with high precision (small standard errors). The AIC and QAIC values for the models for which we assumed that $\lambda$ and $\lambda^{\prime}$ were known are presented in Table 1. According to these values, the two best models were the full model $\left(F_{y}, F_{y}^{\prime}, M_{Y_{-} 91-98}, M_{Y_{-} 99-03}, M_{A_{-} 91-98}, M_{A_{-} 99-03}, \mathrm{Sel}_{3}\right.$, $\mathrm{Sel}_{4}, \mathrm{Sel}_{5}$ ), which had annual values for $F$ and $F^{\prime}$ and the reduced model $\left(F_{91-94}, F_{95-03}, F_{y}^{\prime}, M_{Y \text { 91-98 }}\right.$, $\left.M_{Y \_99-03}, M_{A_{-} 91-98}, M_{A_{-} 99-03}, \mathrm{Sel}_{3}, \mathrm{Sel}_{4}, \mathrm{Sel}_{5}\right)$, in
TABLE 1.-Values of the Akaike information criterion (AIC) and the AIC adjusted for overdispersion (QAIC) for 11 models in which $F$ and $F^{\prime}$ are age-dependent through selectivity. $M$ is constant or assumes age- and/or year-specific values, and $\lambda=\lambda^{\prime}=0.43$. Variables $\Delta \mathrm{AIC}$ and $\Delta \mathrm{QAIC}$ are the differences from the AIC and QAIC values of the best model. The estimated overdispersion is 1.197 , based on model $8 ; K$ is the number of parameters in the model. See text for more details and definitions of variables.

\begin{tabular}{cccrrr}
\hline Model $^{\mathrm{a}}$ & $K$ & \multicolumn{1}{c}{ AIC } & $\Delta$ AIC & QAIC & $\Delta$ QAIC \\
\hline 1 & 30 & $3,637.18$ & 249.66 & $3,040.98$ & 210.72 \\
2 & 31 & $3,528.01$ & 140.49 & $2,950.34$ & 120.08 \\
3 & 33 & $3,546.80$ & 159.28 & $2,966.67$ & 136.40 \\
4 & 31 & $3,502.25$ & 114.73 & $2,928.88$ & 98.61 \\
5 & 31 & $3,542.58$ & 155.06 & $2,962.48$ & 132.22 \\
6 & 31 & $3,511.79$ & 124.27 & $2,936.83$ & 106.56 \\
7 & 31 & $3,511.56$ & 164.04 & $2,969.97$ & 139.70 \\
8 & 33 & $3,395.87$ & 8.35 & $2,840.89$ & 10.63 \\
9 & 22 & $3,387.52$ & 0.00 & $2,830.27$ & 0.00 \\
10 & 21 & $3,464.59$ & 77.07 & $2,894.16$ & 63.89 \\
11 & 21 & $3,432.84$ & 45.32 & $2,867.70$ & 37.43 \\
\hline
\end{tabular}

${ }^{\mathrm{a}}$ The models are as follows:

1: $\left(F_{y}, F, M, \mathrm{Sel}_{3}, \mathrm{Sel}_{4}, \mathrm{Sel}_{5}\right)$

2: $\left(F_{y}, F_{y}, M_{Y}, M_{A}, \mathrm{Sel}_{3}, \mathrm{Sel}_{4}, \mathrm{Sel}_{5}\right)$

3: $\left(F_{y}, F_{y}^{\prime \prime}, M_{3}, M_{4}, M_{5}, M_{6}, \mathrm{Sel}_{3}, \mathrm{Sel}_{4}, \mathrm{Sel}_{5}\right)$

4: $\left(F_{y}, F_{y}^{\prime}, M_{91-98}, M_{99-03}, \mathrm{Sel}_{3}, \mathrm{Sel}_{4}, \mathrm{Sel}_{5}\right)$

5: $\left(F_{y}, F_{y}^{\prime}, M_{91-99}, M_{00-03}, \mathrm{Sel}_{3}, \mathrm{Sel}_{4}, \mathrm{Sel}_{5}\right)$

6: $\left(F_{y}, F_{y}^{\prime}, M_{91-97}, M_{98-03}, \mathrm{Sel}_{3}, \mathrm{Sel}_{4}, \mathrm{Sel}_{5}\right)$

7: $\left(F_{y}, F_{y}^{\prime}, M_{91-96}, M_{97-03}, \mathrm{Sel}_{3}, \mathrm{Sel}_{4}, \mathrm{Sel}_{5}\right)$

8: $\left(F_{y}, F_{y}^{\prime}, M_{Y_{-} 91-98}, M_{Y_{-} 99-03}, M_{A_{-91-98}}, M_{A_{9} 99-03}, \mathrm{Sel}_{3}, \mathrm{Sel}_{4}, \mathrm{Sel}_{5}\right)$

9: $\left(F_{91-94}, F_{95-03}, F_{y}^{\prime}, \bar{M}_{Y_{-} 91-98}, \bar{M}_{Y_{-} 99-03}, \bar{M}_{A_{-} 91-98}, M_{A_{-} 99-03}, \mathrm{Sel}_{3}\right.$, $\mathrm{Sel}_{4}, \mathrm{Sel}_{5}$ )

10: $\left(F_{y}, F_{y}^{\prime}, M_{Y_{-} 91-98}, M_{Y_{Y} 99-03}, M_{A_{-} 91-98}, M_{A_{-} 99-03}, \mathrm{Sel}_{3}, \mathrm{Sel}_{4}, \mathrm{Sel}_{5}\right)$ 11: $\left(F, F_{y}^{\prime}, M_{Y_{-} 91-98}, M_{Y_{-} 99-03}, M_{A_{-} 91-98}^{A_{-} 91-98}, M_{A_{-}-99-03}^{A_{-} 99-03}, \mathrm{Sel}_{3}, \mathrm{Sel}_{4}, \mathrm{Sel}_{5}\right)$.

which $F$ was constant in the years before (1991-1994) and after (1995-2003) a regulation change. Both of the best models allowed the natural mortality rate, $M$, to vary by age and year. There was little difference between the AIC and QAIC criteria because the value of the inflation factor (1.20) was very close to 1.0. There was thus little evidence of overdispersion in this example. These models assumed that $M$ was different for two age-groups, young (age 3 to age 5) and adult (age 6 and above), and for two time periods, 1991 to 1998 and 1999 to 2003. In combination, we had four different natural mortality rates to estimate.

Based on the AIC and QAIC values for models 2-7 (Table 1), we focused on models in which natural mortality was different for young and adult fish and/or for the periods 1991-1998 and 1999-2003. Of all the models in Table 1, the one with a constant value of $M$ has the worst fit. Comparing the two best models using a likelihood ratio test results in $\chi^{2}=14$ with $11 \mathrm{df}(P=$ $0.23)$, suggesting that the reduced model ( $F_{91-94}$, $F_{95-03}, F_{y}^{\prime}, M_{Y_{-} 91-98}, M_{Y_{-} 99-03}, M_{A_{-} 91-98}, M_{A_{-} 99-03}$, $\left.\mathrm{Sel}_{3}, \mathrm{Sel}_{4}, \mathrm{Sel}_{5}\right)$ is preferred over the more general model $\left(F_{y}, F_{y}^{\prime}, M_{Y_{-} 91-98}, M_{Y_{-} 99-03}, M_{A_{-} 91-98}, M_{A_{-} 99-03}\right.$, 
$\mathrm{Sel}_{3}, \mathrm{Sel}_{4}, \mathrm{Sel}_{5}$ ) (which agrees with the QAIC criterion) and that the variation in fishing mortality is mainly associated with an increase in mortality after the relaxation of fishing regulations.

Parameter estimates for both models are shown in Table 2. Both models result in estimates with good precision; the relative standard errors for most parameter estimates are less than $10 \%$. As might be expected, the estimated precision for fishing mortality rates is better in the reduced model. Selectivity estimates have the expected trend in both models, with an estimate of about 1.0 for fish of age 5. In the reduced model, the estimated natural mortality for young fish is 0.40 ( $\mathrm{SE}=0.02$ ) from 1991 to 1998 , which is higher than that for adults $(0.15 ; \mathrm{SE}=0.01)$. From 1999 to 2003, the estimated natural mortality for young fish is $0.86(\mathrm{SE}=0.06)$, which is higher than that for adults $(0.65 ; \mathrm{SE}=0.03)$. The estimates also indicate that, for both young and adult striped bass, the natural mortality rate is lower in the earlier years than in the later years and that fishing mortality increased after fishing regulations were relaxed in 1995.

For the models investigated in Tables 1 and 2, we assumed that $\lambda=\lambda^{\prime}=0.43$ based on the best information available on reporting rates. If the true values of $\lambda$ and $\lambda^{\prime}$ are not 0.43 , we will obtain biased parameter estimates. The increase in the estimates of $M$ in later years could actually be due to a decrease in $\lambda$ and $\lambda^{\prime}$, and we investigated that alternative. However, $\lambda$ and $\lambda^{\prime}$ would have had to decrease to values less than 0.20 for the estimates of $M$ to remain the same, and we believe this very unlikely. Thus, the evidence for an increase in $M$ over time is robust, but the magnitude of the increase is tied to the values of the tag reporting rate. This emphasizes the importance of obtaining accurate, independent information about reporting rates.

Models in which $\lambda$ and $\lambda^{\prime}$ were estimated were also considered (Table 3$)$. The general model $\left(F_{y}, F_{y}^{\prime}\right.$, $M_{Y_{-} 91-98}, M_{Y_{-} 99-03}, M_{A_{-} 91-98}, M_{A_{-} 99-03}, \mathrm{Sel}_{3}, \mathrm{Sel}_{4}$, $\mathrm{Sel}_{5}, \lambda, \lambda^{\prime}$ ) produced reasonable point estimates but generally had low precision except for the selectivity estimates. It is very interesting that the estimates of $\lambda$ and $\lambda^{\prime}$ ( 0.41 and 0.43 , respectively) agree well with the common value of 0.43 assumed in the previous models. The reduced model $\left(F_{91-94}, F_{95-03}, F_{y}^{\prime}, M_{Y_{-91-98}}\right.$, $\left.M_{Y_{-} 99-03}, M_{A_{-} 91-98}, M_{A_{-} 99-03}, \mathrm{Sel}_{3}, \mathrm{Sel}_{4}, \mathrm{Sel}_{5}, \bar{\lambda}, \lambda^{\prime}\right)$ produced some unreasonable estimates, and again the estimates generally have low precision. In particular, adult natural mortality from 1991 to 1998 was estimated as 0.001 , which is not at all reasonable. Estimates obtained with the models in which reporting rates were estimated are sensitive to the initial values of the parameters used in the estimation process. The poor
TABLE 2.-Parameter estimates (SEs in parentheses) from fitting catch-and-release tag return models allowing age- and year-specific natural mortality and selectivity for fishing mortality to Chesapeake Bay striped bass data. Model (a) $\left(F_{y}\right.$, $F_{y}^{\prime}, M_{Y-91-98}, M_{Y_{-} 99-03}, M_{A_{-91-98}}, M_{A_{-} 99-03}, \mathrm{Sel}_{3}, \mathrm{Sel}_{4}, \mathrm{Sel}_{5}$ ) allows fishing mortality to vary by year and Model (b) $\left(F_{91-94}\right.$, $F_{95-03}, F_{y}^{\prime}, M_{Y \_91-98}, M_{Y_{-99-03}}, M_{A_{-91-98}}, M_{A \_99-03}, \mathrm{Sel}_{3}, \mathrm{Sel}_{4}$, $\mathrm{Sel}_{5}$ ) makes fishing mortality constant (at different rates) before and after 1995, when fishing regulations were liberalized. Reporting rates for harvested and released fish were fixed at 0.43 .

\begin{tabular}{|c|c|c|}
\hline \multirow[b]{2}{*}{ Parameter $^{\mathrm{a}}$} & \multicolumn{2}{|c|}{ Model } \\
\hline & (a) & (b) \\
\hline$F_{91}$ & $0.106(0.014)$ & $0.154(0.007)$ \\
\hline$F_{92}^{91}$ & $0.163(0.014)$ & $0.154(0.007)$ \\
\hline$F_{93}^{92}$ & $0.152(0.011)$ & $0.154(0.007)$ \\
\hline$F_{94}^{93}$ & $0.162(0.011)$ & $0.154(0.007)$ \\
\hline$F_{95}^{94}$ & $0.226(0.013)$ & $0.235(0.007)$ \\
\hline$F_{96}$ & $0.190(0.012)$ & $0.235(0.007)$ \\
\hline$F_{97}^{96}$ & $0.233(0.015)$ & $0.235(0.007)$ \\
\hline$F_{98}^{9 \prime}$ & $0.244(0.017)$ & $0.235(0.007)$ \\
\hline$F_{99}^{98}$ & $0.254(0.019)$ & $0.235(0.007)$ \\
\hline$F_{00}$ & $0.260(0.018)$ & $0.235(0.007)$ \\
\hline$F_{01}$ & $0.293(0.022)$ & $0.235(0.007)$ \\
\hline$F_{02}$ & $0.230(0.018)$ & $0.235(0.007)$ \\
\hline$F_{03}^{02}$ & $0.140(0.022)$ & $0.235(0.007)$ \\
\hline$F_{91}^{\prime 3}$ & $0.125(0.016)$ & $0.124(0.016)$ \\
\hline$F_{92}^{\prime}$ & $0.156(0.013)$ & $0.160(0.014)$ \\
\hline$F_{93}^{\prime \prime}$ & $0.105(0.009)$ & $0.109(0.009)$ \\
\hline$F_{94}^{\prime}$ & $0.132(0.010)$ & $0.131(0.010)$ \\
\hline$F_{95}^{\prime}$ & $0.106(0.009)$ & $0.117(0.009)$ \\
\hline$F_{96}^{\prime \prime}$ & $0.116(0.009)$ & $0.125(0.010)$ \\
\hline$F_{97}^{\prime}$ & $0.092(0.009)$ & $0.099(0.009)$ \\
\hline$F_{98}^{\prime \prime}$ & $0.094(0.010)$ & $0.095(0.010)$ \\
\hline$F_{99}^{\prime}$ & $0.074(0.010)$ & $0.082(0.010)$ \\
\hline$F_{00}^{\prime \prime}$ & $0.169(0.014)$ & $0.168(0.014)$ \\
\hline$F_{01}^{\prime}$ & $0.126(0.013)$ & $0.123(0.012)$ \\
\hline$F_{02}^{\prime 1}$ & $0.081(0.009)$ & $0.092(0.009)$ \\
\hline$F_{03}^{\prime}$ & $0.056(0.012)$ & $0.050(0.011)$ \\
\hline $\mathrm{Sel}_{3}$ & $0.663(0.061)$ & $0.627(0.058)$ \\
\hline $\mathrm{Sel}_{4}$ & $0.730(0.044)$ & $0.739(0.044)$ \\
\hline $\mathrm{Sel}_{5}^{4}$ & $0.967(0.047)$ & $1.000(0.048)$ \\
\hline$M_{Y-91-98}$ & $0.378(0.021)$ & $0.399(0.021)$ \\
\hline$M_{Y}-99-03$ & $0.836(0.063)$ & $0.858(0.056)$ \\
\hline$M_{Y 91-98}^{Y_{-99-03}}$ & $0.145(0.009)$ & $0.150(0.009)$ \\
\hline$M_{Y_{-} 99-03}^{Y_{-9198}}$ & $0.673(0.038)$ & $0.645(0.028)$ \\
\hline
\end{tabular}

${ }^{a}$ Two-digit subscripts refer to individual years; those with dashes refer to ranges of years.

performance of the models with estimated reporting rates suggested that we use external sources such as special high-reward tagging studies to obtain accurate information on reporting rates.

Recall that the estimates of $F_{y}^{\prime}$ in Tables 2 and 3 represent a mortality force on the tags removed from fish that were released. To calculate estimates of total mortality for fish, we account for the mortality of fish that were released by adjusting the estimate of $F_{y}^{\prime}$ using an estimate of hooking mortality as described for the age-independent case. For example, assuming that $\lambda=$ $\lambda^{\prime}=0.43$ and that hooking mortality $\delta=0.09$, in the 
TABLE 3.-Parameter estimates (SEs in parentheses) from fitting catch-and-release tag return models allowing age- and year-specific natural mortality and selectivity for fishing mortality to Chesapeake Bay striped bass data. The reporting rates $\left(\lambda\right.$ and $\left.\lambda^{\prime}\right)$ are also estimated. Models (a) and (b) are identical to those in Table 2 except for the inclusion of $\lambda$ and $\lambda^{\prime}$ as variables.

\begin{tabular}{|c|c|c|}
\hline \multirow[b]{2}{*}{ Parameter } & \multicolumn{2}{|c|}{ Model } \\
\hline & (a) & (b) \\
\hline$F_{91}$ & $0.173(0.075)$ & $0.208(0.053)$ \\
\hline$F_{02}^{91}$ & $0.185(0.080)$ & $0.208(0.053)$ \\
\hline$F_{93}$ & $0.164(0.071)$ & $0.208(0.053)$ \\
\hline$F_{94}^{93}$ & $0.144(0.059)$ & $0.208(0.053)$ \\
\hline$F_{95}^{94}$ & $0.269(0.110)$ & $0.315(0.080)$ \\
\hline$F_{93}$ & $0.207(0.086)$ & $0.315(0.080)$ \\
\hline$F_{97}^{96}$ & $0.238(0.100)$ & $0.315(0.080)$ \\
\hline$F_{98}^{9 \prime}$ & $0.288(0.125)$ & $0.315(0.080)$ \\
\hline$F_{99}$ & $0.264(0.116)$ & $0.315(0.080)$ \\
\hline$F_{00}$ & $0.236(0.102)$ & $0.315(0.080)$ \\
\hline$F_{01}^{00}$ & $0.228(0.096)$ & $0.315(0.080)$ \\
\hline$F_{02}$ & $0.173(0.069)$ & $0.315(0.080)$ \\
\hline$F_{03}$ & $0.108(0.036)$ & $0.315(0.080)$ \\
\hline$F_{91}^{\prime \prime}$ & $0.157(0.150)$ & $0.204(0.091)$ \\
\hline$F_{92}^{\prime}$ & $0.158(0.153)$ & $0.268(0.118)$ \\
\hline$F_{93}^{\prime \prime}$ & $0.119(0.112)$ & $0.184(0.082)$ \\
\hline$F_{94}^{\prime}$ & $0.122(0.116)$ & $0.216(0.094)$ \\
\hline$F_{95}^{\prime \prime}$ & $0.121(0.115)$ & $0.193(0.085)$ \\
\hline$F_{96}^{\prime}$ & $0.116(0.110)$ & $0.206(0.092)$ \\
\hline$F_{97}^{\prime}$ & $0.114(0.109)$ & $0.161(0.073)$ \\
\hline$F_{98}^{\prime \prime}$ & $0.104(0.099)$ & $0.156(0.070)$ \\
\hline$F_{99}^{\prime}$ & $0.081(0.077)$ & $0.130(0.058)$ \\
\hline$F_{00}^{\prime}$ & $0.125(0.120)$ & $0.275(0.121)$ \\
\hline$F_{01}^{\prime}$ & $0.089(0.085)$ & $0.203(0.092)$ \\
\hline$F_{02}^{\prime \prime}$ & $0.068(0.063)$ & $0.156(0.070)$ \\
\hline$F_{03}^{\prime}$ & $0.035(0.030)$ & $0.083(0.039)$ \\
\hline$\lambda^{03}$ & $0.407(0.170)$ & $0.321(0.081)$ \\
\hline$\lambda^{\prime}$ & $0.430(0.407)$ & $0.261(0.113)$ \\
\hline $\mathrm{Sel}_{3}$ & $0.755(0.066)$ & $0.621(0.059)$ \\
\hline $\mathrm{Sel}_{4}^{3}$ & $0.810(0.049)$ & $0.726(0.045)$ \\
\hline $\mathrm{Sel}_{5}^{4}$ & $0.978(0.048)$ & $1.000(0.047)$ \\
\hline$M_{Y-91-98}$ & $0.405(0.159)$ & $0.283(0.110)$ \\
\hline$M_{Y}^{Y_{-}-91-98}$ & $0.678(0.170)$ & $0.726(0.132)$ \\
\hline$M_{Y-91-98}^{Y-99-03}$ & $0.148(0.183)$ & $0.001(0.134)$ \\
\hline$M_{Y_{-} 99-03}^{T_{-91-98}}$ & $0.480(0.185)$ & $0.491(0.146)$ \\
\hline
\end{tabular}

reduced model in Table 2 we calculate total mortality for adult fish in 1991 as

$$
\begin{aligned}
\hat{Z}_{A \_91-94, \text { fish }} & =\hat{F}_{91-94}+\delta \cdot \hat{F}_{91}^{\prime}+\hat{M}_{A \_91-98} \\
& =0.154+(0.09)(0.124)+0.15=0.315 .
\end{aligned}
$$

The annual survival rate for adult fish in 1991 is then estimated to be $e^{-0.315}=0.73$.

\section{Discussion}

Different approaches exist for estimating survival and mortality rates from tag return data where tagged fish are subject to harvest as well as catch and release. Youngs and Robson (1975) did not include fish that were released alive in their analysis of data for lake trout Salvelinus namaycush. Burnham (1993) and Barker (1997) developed methods for the joint analysis of data from fish that were harvested or caught and released. These two methods assume that tags are not removed prior to release and that the catch-and-release procedure does not affect survival. However, for the Maryland striped bass study, tags were removed (regardless of the disposition of the recaptured fish) prior to reporting the tag numbers to the U.S. Fish and Wildlife Service. High proportions $(36 \%$ for all fish, $32 \%$ for adult fish) of striped bass tags were reported from caught-and-released fish. Also, the catch-andrelease procedure affects survival (Diodati and Richards 1996; Millard et al. 2003). Thus, the Youngs and Robson (1975), Burnham (1993), and Barker (1997) approaches are not valid for the Maryland striped bass study.

Smith et al. (2000) developed models to estimate the finite rates of survival and recovery from catch-andrelease data wherein high proportions of tags were reported from caught-and-released fish, adjusting the bias caused by fish released alive with tag removed before reporting to the fishery agency. In their somewhat ad hoc approach, they assumed that the natural mortality rate is 0.15 , that reporting rates are constant and known, and that all parameters are age independent. In contrast, our models assume that the reporting rates are constant and known and allow age and year dependence for $M$. Our models also allow for estimation of the reporting rates and the incorporation of additional components of the likelihood function to account for supplemental data relating to the tag reporting rate so as to obtain estimates with good precision.

We extend the instantaneous rate formulation of tag return models to analyze the catch-and-release study data. Despite its great importance to stock assessments, the natural mortality rate is difficult to estimate for all fish species (Vetter 1988; Hoenig et al. 1998a) and for striped bass in particular (Hightower et al. 2001). One very important feature of our models is that a limited degree of age and/or year dependence can be allowed in the estimation of natural mortality. Analyses of the Chesapeake Bay striped bass data from Maryland demonstrate that models with a limited degree of ageand year-dependent natural mortality are strongly preferred to models with more restrictive assumptions about $M$. For both young and adult fish, our results show that total mortality increased and annual survival decreased after 1995, which probably reflect the change in harvest regulations and the effect of disease.

One very important result for fisheries managers is that we provide some of the first empirical evidence that $M$ has increased in recent years for the Maryland Chesapeake Bay population of striped bass. This may be due to the emergence of mycobacteriosis in this 
population in the late 1990s (Cardinal 2001). A large drop in the reporting rate (to less than 20\%) is an alternative explanation, but one we believe to be extremely unlikely.

We attempted to address the issue of uncertainty about reporting rates directly by estimating them internal to our analysis. However, owing to parameter redundancy problems, we found that models in which tag reporting rates were estimated produced some unrealistic estimates and that even the realistic estimates had low precision. Thus the importance of estimating tag reporting rates from special external information such as high-reward tagging studies or possibly angler surveys (Pollock et al. 2001, 2002; Hearn et al. 2003) is obvious. This is clearly an important area for future research, and in particular we recommend that special, ongoing high-reward tagging studies be initiated to obtain better estimates of the reporting rates for harvested and released fish. This would also enable scientists to empirically examine the critical assumptions that these two reporting rates are equal, constant over time, and constant over fish ages. The methods used by Smith et al. (2000) also made these critical assumptions about reporting rates.

The approach developed here assumes that fish are correctly assigned to age categories, either by examining some hard structure such as a scale or through the use of an age-length key. Even if age assignments are unbiased, there will be some level of misclassification using either method. These misclassifications could result in some bias in model estimates if parameter vary substantially among ages. For the Chesapeake Bay striped bass example, there were moderate differences in estimated selectivity across ages $3-5$, but natural mortality was quite different for the young (age 3-5) and adult (age 6+) age categories. The potential for bias when hard parts are used to assign fish to age-classes can be assessed through age validation studies (Beamish and McFarlane 1983). When an age-length key is used, the overlap in length distributions among ages provides some indication of the potential bias.

For the Maryland striped bass study, tags were removed from the fish before they were released alive. In studies in which tags are not cut off before release, the marked fish can be recaptured multiple times and additional information on survival can be obtained. For future research, the generalized Jolly-Seber model (Seber 1982; Williams et al. 2002) can be applied to the case where the tags of fish released alive are not cut off.

\section{Acknowledgments}

Support for this work was provided by WallopBreaux funds from the U.S. Fish and Wildlife Service under contract F-77-19 through the Virginia Marine
Resources Commission and by Anadromous Fish Conservation Act program funds from the National Marine Fisheries Service under grant NA05NMF4051116. Additional funds were provided by the Virginia Marine Resources Commission Recreational Fishing Advisory Board. We thank the field collection staff with the Maryland Department of Natural Resources. We also thank Tina McCrobie of the U.S. Fish and Wildlife Service for help with assembling the data set. Bill Hearn provided helpful comments on an earlier version of the manuscript, and Barbara Brunhuber provided editorial assistance.

\section{References}

Barker, R. J. 1997. Joint modeling of live-recapture, tagresight, and tag recovery data. Biometrics 53:666-677.

Beamish, R. J., and G. A. McFarlane. 1983. The forgotten requirement for age validation in fisheries biology. Transactions of the American Fisheries Society 112:735-743.

Brooks, E. N., K. H. Pollock, J. M. Hoenig, and W. S. Hearn. 1998. Estimation of fishing and natural mortality from tagging studies on fisheries with two user groups. Canadian Journal of Fisheries and Aquatic Sciences 55:2001-2010.

Burnham, K. P. 1993. A theory for combined analysis of ring recovery and recapture data. Pages 199-214 in J. D. Lebreton and P. M. North, editors. Marked individuals in the study of bird population. Birkhauser Verlag, Basel, Switzerland.

Burnham, K. P., and D. R. Anderson. 2002. Model selection and inference: a practical information-theoretic approach, 2nd edition. Springer-Verlag, New York.

Cardinal, J. L. 2001. Mycobacteriosis in striped bass, Morone saxatilis, from Virginia waters of Chesapeake Bay. Master's thesis. Virginia Institute of Marine Science, Gloucester Point.

Diodati, P. J., and A. R. Richards. 1996. Mortality of striped bass hooked and released in salt water. Transactions of the American Fisheries Society 125:300-307.

Hearn, W. S., J. M. Hoenig, K. H. Pollock, and D. A. Hepworth. 2003. Tag reporting rate estimation, 3. Use of planted tags in one component of a multicomponent fishery. North American Journal of Fisheries Management 23:66-77.

Hightower, J. E., J. R. Jackson, and K. H. Pollock. 2001. Using telemetry methods to estimate natural and fishing mortality of striped bass in Lake Gaston, North Carolina. Transactions of the American Fisheries Society 130:557567.

Hoenig, J. M., N. J. Barrowman, W. S. Hearn, and K. H. Pollock. 1998a. Multiyear tagging studies incorporating fishing effort data. Canadian Journal of Fisheries and Aquatic Sciences 55:1466-1476.

Hoenig, J. M., N. J. Barrowman, K. H. Pollock, E. N. Brooks, W. S. Hearn, and T. Polacheck. 1998b. Models for tagging data that allow for incomplete mixing of newly tagged animals. Canadian Journal of Fisheries and Aquatic Sciences 55:1477-1483.

Jiang, H. 2005. Age-dependent tag return models for 
estimating fishing mortality, natural mortality, and selectivity. Doctoral dissertation. North Carolina State University, Raleigh.

Millard, M. J., S. A. Welch, J. W. Fletcher, J. Mohler, A. Kahnle, and K. Hattala. 2003. Mortality associated with catch and release of striped bass in the Hudson River. Fisheries Management and Ecology 10:295-300.

Pollock, K. H., J. M. Hoenig, W. S. Hearn, and B. Calingaert. 2001. Tag reporting rate estimation, 1. An evaluation of the high-reward tagging method. North American Journal of Fisheries Management 21:521-532.

Pollock, K. H., J. M. Hoenig, W. S. Hearn, and B. Calingaert. 2002. Tag reporting rate estimation, 2. Use of highreward tagging and observers in multicomponent fisheries. North American Journal of Fisheries Management 22:727-736

Pollock, K. H., J. M. Hoenig, and C. M. Jones. 1991. Estimation of fishing and natural mortality when a tagging study is combined with a creel survey or port sampling. Pages 423-434 in D. Guthrie, J. M. Hoenig, M. Holliday, C. M. Jones, M. J. Mills, S. A. Moberley, K. H. Pollock, and D. R. Talhelm, editors. Creel and angler surveys in fisheries management. American Fisheries Society, Symposium 12, Bethesda, Maryland.

Richards, A. R., and P. J. Rago. 1999. A case history of effective fishery management: Chesapeake Bay striped bass. North American Journal of Fisheries Management 19:356-375.
Seber, G. A. F. 1982. The estimation of animal abundance and related parameters, 2nd edition. Charles W. Griffin, London.

Secor, D. H., T. M. Trice, and H. T. Hornick. 1995. Validation of otolith-based aging and a comparison of otolith- and scale-based aging in mark-harvested Chesapeake Bay striped bass, Morone saxatilis. U.S. National Marine Fisheries Service Fishery Bulletin 93:186-190.

Smith, D. R., K. P. Burnham, D. M. Kahn, X. He, G. J. Goshorn, K. A. Hattala, and A. W. Kahnle. 2000. Bias in survival estimates from tag recovery models where catch and release is common, with an example from Atlantic striped bass (Morone saxatilis). Canadian Journal of Fisheries and Aquatic Sciences 57:886-897.

Vetter, E. F. 1988. Estimation of natural mortality in fish stocks: a review. U.S. National Marine Fisheries Service Fishery Bulletin 86:25-43.

White, G. C. 1983. Numerical estimation of survival rates from band-recovery and biotelemetry data. Journal of Wildlife Management 47:716-728.

Williams, B. K., J. D. Nichols, and M. J. Conroy. 2002. Analysis and management of animal populations. Academic Press, San Diego, California.

Youngs, W. D., and D. S. Robson. 1975. Estimating survival rates from tag returns: model tests and sample size determination. Journal of the Fisheries Research Board of Canada 32:2365-2371. 